\title{
Experiencias en la atención en salud de mujeres con anormalidades citológicas o cáncer de cuello uterino en dos departamentos fronterizos: una lucha entre la vida y el sistema de salud colombiano
}

\author{
Experiences in the Health Care of Women with Cytological Abnormalities or Cervix \\ Cancer in Two Border Departments: A Struggle between Life and the Colombian \\ Health System
}

\section{Experiências na atenção em saúde de mulheres com anormalidades citológicas ou câncer de colo de útero em dois estados da fronteira: uma luta entre a vida e o sistema de saúde colombiano}

\author{
Diana Patricia Molina-Berrío'; Sara Milena Ramos-Jaraba²; Isabel C Garcés-Palacio³ \\ Magíster en Salud Pública. Universidad de Antioquia, Medellín, Colombia. dpatricia.molina@udea.edu.co. ORCID 0000-0002-1352-1089 \\ Magíster en Salud Colectiva. Universidad de Antioquia, Medellín, Colombia. sara.ramos@udea.edu.co. ORCID 0000-0002-8638-5209 \\ 3 Doctora y Magíster en Salud Pública. Universidad de Antioquia, Medellín, Colombia. icristina.garces@udea.edu.co. ORCID 0000- \\ 0003-0531-276X
}

Recibido: 28/04/2020. Aprobado: 16/12/2020. Publicado: 04/03/2021

Molina-Berrío DP, Ramos-Jaraba SM, Garcés-Palacio IC. Experiencias en la atención en salud de mujeres con anormalidades citológicas o cáncer de cuello uterino en dos departamentos fronterizos: una lucha entre la vida y el sistema de salud colombiano. Rev. Fac. Nac. Salud Pública. 2021;39(2):e341399. Dor: https://doi.org/10.17533/udea.rfnsp.e341399

\section{Resumen}

Objetivo: Describir la experiencia en la atención en salud de mujeres con anormalidades citológicas con biopsia negativa para malignidad o cáncer de cuello uterino invasor no mayor a 2 meses, en los departamentos de La Guajira y Nariño, en Colombia, entre 2016 y 2017. Metodología: Se presenta el componente cualitativo de un estudio de métodos mixtos de triangulación convergente, a través de 10 entrevistas y 3 grupos focales. Participaron 9 mujeres con cáncer y 6 con anormalidades citológicas entre los 43 y 65 años de edad, 6 de ellas indígenas y 14 pertenecientes al régimen subsidiado. Resultados: Las participantes mencionaron experiencias negativas frente a la atención en salud, pues enfrentan múltiples barreras para acceder al diagnóstico y al tratamiento, como demora en la asignación y la autorización de citas, exámenes y procedimientos; el gasto de bolsillo para traslados a ciudades capitales donde se brinda el servicio; dificultades para encontrar acompañamiento familiar fuera de sus comunidades de origen y barreras idiomáticas en mujeres de comunidades indígenas. También se presentaron dificultades de comunicación efectiva con el personal de salud, lo que llevó a algunas participantes a no entender su diagnóstico 
o tratamiento, y a no sentirse escuchadas. La vivencia del cáncer generó sufrimiento, rechazo de personas de su entorno social y despersonalización. Conclusiones: Las barreras de diversa índole para el acceso oportuno al tratamiento se agudizan por características de base como la pobreza y la pertenencia a grupos étnicos, convirtiéndose la supervivencia en una lucha diaria, que va más allá de las características propias de la enfermedad.

Palabras clave: Salud de la mujer, neoplasias del cuello uterino, poblaciones vulnerables, barreras de acceso a los servicios de salud, interseccionalidad, La Guajira, Nariño, Colombia.

\section{Abstract}

Objective: To describe the health care experience of women with cytological abnormalities with negative biopsy for malignancy or invasive cervix cancer within two months after diagnosis, in La Guajira and Nariño departments, Colombia, between 2016 and 2017. Methodology: The qualitative component of a mixed methods study of convergent triangulation is presented, through ten interviews and three focus groups. Nine women with cancer and six women with cytological abnormalities between 43 and 65 years of age participated; six of them were indigenous, and fourteen are enrolled to the subsidized health regime. Results: Participants referred negative experiences in the health care system as they face numerous barriers for the diagnosis and access to treatment. Such as, delay in the assignment and authorization of medical appointments, tests, and procedures; the out-of-pocket expenses for transportation to capital cities where the health service is provided; difficulties to find family support outside their communities of origin, and language barriers among women from indigenous communities. Besides, effective communication problems with health personnel were also presented, which lead some participants to misunderstanding their diagnosis or treatment and feeling that they were not being heard. The experience of having cancer led to suffering, rejection from people in their social environment, and depersonalization. Conclusions: Barriers of various kinds for the timely access to treatment are exacerbated by baseline characteristics such as poverty and belonging to ethnic groups, turning survival into a daily struggle, which goes beyond the specific characteristics of the disease itself.

-Key words: women's health, uterine cervical neoplasms, vulnerable populations, barriers to access health services, cross-sectionality, La Guajira, Nariño, Colombia.

\section{Resumo}

Objetivo: Descrever a experiência na atenção em saúde de mulheres com anormalidades citológicas com biopsia negativa para malignidade ou câncer de colo de útero invasivo não maior a 2 meses nos estados de La Guajira e Nariño, na Colômbia, entre 2016 e 2017. Metodologia: Foi apresentado o componente qualitativo de um estudo de métodos mistos de triangulação convergente, através de 10 entrevistas e 3 grupos de foco. Participaram 9 mulheres com câncer e 6 com anormalidades citológicas entre 43 e 65 anos da idade, 6 delas indígenas e 14 pertencentes ao sistema subsidiado. Resultados: As participantes mencionaram experiências negativas na atenção em saúde, pois enfrentaram múltiplos obstáculos para ter acesso ao diagnóstico e ao tratamento, como demora na marcação e autorização de consultas, exames e procedimentos; despesas com transporte para cidades capitais onde se oferece o serviço; dificuldades para ter acompanhamento familiar fora das suas comunidades de origem e barreiras idiomáticas para mulheres de comunidades indígenas. Apresentaram-se também, dificuldades de comunicação efetiva com o pessoal de saúde, o que levou a algumas participantes a não entender seu diagnóstico ou tratamento e a não se sentirem escutadas. A experiência do câncer gerou sofrimento, rejeição de pessoas do entorno social e despersonalização. Conclusões: As barreiras de diversas índoles para o acesso oportuno ao tratamento foram agudizadas por características de base como a pobreza e ao fato de pertencer a grupos étnicos, transformando a sobrevivência numa luta diária, que vai além das características próprias da enfermidade.

--------Palavras-chave: Saúde da mulher; neoplastias de colo de útero; populações vulneráveis, barreiras de acesso aos serviços de saúde; interseccionalidade, La Guajira, Nariño, Colômbia 


\section{Introducción}

El cáncer de cuello uterino $(\mathrm{CaCu})$ es el cuarto tipo de cáncer más común en las mujeres a nivel mundial, con un estimado de 570000 casos nuevos en 2018, lo que representa el 6,6\% de todos los cánceres femeninos [1]. Se estima que el $85 \%$ de la carga global se concentra en países de bajos y medianos ingresos, pues 9 de cada 10 $(87 \%)$ muertes ocurren en regiones menos desarrolladas, con las tasas de incidencia y mortalidad más altas en África, América Latina y el Caribe [2]. En Colombia, el $\mathrm{CaCu}$ representa la segunda causa de muerte por cáncer entre las mujeres, seguido del cáncer de mama [3].

$\mathrm{El} \mathrm{CaCu}$ es fácil de prevenir y altamente curable cuando se detecta y se trata en estadios tempranos, por lo que en el país se han desarrollado estrategias para mejorar la oportunidad en la prevención, el diagnóstico y el tratamiento del cáncer. Entre ellas se destaca la promulgación de la Ley 1384 de 2010 (Ley Sandra Ceballos) [4], el "Plan nacional para el control del cáncer 2012-2021" [5] y el "Modelo para el control del cáncer en Colombia" [6], con los cuales se pretende dar respuesta, de manera intersectorial e integral, a la prevención, el diagnóstico, el tratamiento, la rehabilitación y el cuidado paliativo del cáncer, mejorar la calidad de vida de las personas que viven con cáncer y articular las acciones de los actores del Sistema General de Seguridad Social en Salud [7, p. 8]. A pesar de estos esfuerzos, en 2018 se presentaron 2128 casos nuevos de $\mathrm{CaCu}$, de los cuales 1385 fueron clasificados como invasivos y 1002 mujeres murieron [8]; es decir, más de la mitad de los casos que se detectan ya se encuentran en estadios avanzados, en los que la probabilidad de supervivencia disminuye considerablemente.

La combinación de factores de tipo social, económico, político y cultural ha contribuido a que en el país no se logre una disminución sustancial de la mortalidad por este cáncer, pues se reportan múltiples barreras del sistema de salud, como la distancia geográfica, los problemas en la calidad de la citología, la fragmentación en la prestación de los servicios de salud (que, en el caso particular, se evidencia en que los servicios de atención, diagnóstico y tratamiento del cáncer son brindados por diferentes prestadores de servicios de salud), los problemas de tipo administrativo, y la falta de sensibilidad y adecuación cultural de los servicios [9-11]. Existen también demoras para iniciar un tratamiento oportuno: los hallazgos de algunos estudios reportan que las mujeres más pobres, afiliadas al régimen subsidiado, o que no tienen seguro, y que viven en zonas apartadas inician su tratamiento cuando el cáncer está avanzado, y que, además, deben desplazarse a otros departamentos a recibir atención en salud, lo que dificulta la adherencia [12-14]. Esto último da cuenta de la existencia de mercados de salud concentrados, pues según el Instituto Nacional de Cancerología, el 57,6\% de los servicios oncológicos del país se hallan en ciudades capitales (Barranquilla, Bogotá, Cali, Cartagena y Medellín) [15], lo que impide el acceso efectivo al tratamiento de los pacientes oncológicos de lugares apartados del país, entre los cuales se encuentran los departamentos fronterizos, como La Guajira y Nariño. Según una investigación realizada en La Guajira, aunque en la regulación del sistema de salud colombiano se tienen en cuenta las especificidades para la prestación de servicios a las comunidades indígenas, la implementación de esta es limitada [16].

Sumado a lo anterior, también existen aspectos socioculturales que no han logrado ser tenidos en cuenta en los programas de prevención y tratamiento de $\mathrm{CaCu}$. En las mujeres de diversas etnias y regiones se presentan barreras culturales asociadas a la interpretación errada del proceso de la enfermedad, miedo al estigma por el diagnóstico, desconocimiento de los efectos adversos de los tratamientos, temor, pudor, poco apoyo familiar, dificultades económicas y barreras idiomáticas que influyen de manera importante y dificultan el acceso a los servicios de salud [17-19]. En una muestra de mujeres de varios departamentos de Colombia, Wiesner et al. encontraron que el $27 \%$ de las mujeres con lesiones de alto grado o invasoras no tuvieron acceso a diagnóstico o tratamiento, debido a obstáculos administrativos, razones clínicas o culturales [20]. Aunado a estas barreras, la experiencia del cáncer trae consigo modificaciones en todos los ámbitos de vida de las mujeres, lo que afecta no solo su salud física, sino también la mental, las relaciones familiares, sentimentales y su economía, siendo este proceso más complejo para aquellas en condiciones precarias que deben trasladarse fuera de su entorno social y cultural para recibir tratamiento [13].

Teniendo en cuenta que el proceso salud-enfermedad-atención opera de manera diferenciada en los diversos grupos sociales, ya sea que se delimite una problemática particular o que se centre en un contexto particular, y que, además, por la complejidad del sistema de salud, los programas de prevención de $\mathrm{CaCu}$ presentan limitaciones para disminuir la mortalidad de este cáncer, especialmente en minorías étnicas y departamentos fronterizos, esta investigación brinda elementos adicionales que permiten comprender las experiencias en la atención en salud de mujeres con anormalidades citológicas o $\mathrm{CaCu}$ invasor en los departamentos de La Guajira y Nariño en Colombia, con el fin de contribuir a una mejor compresión de la problemática de estos lugares y comunidades.

La pregunta sobre la experiencia de las mujeres frente a la atención recibida cuando se tiene una anormalidad citológica o un $\mathrm{CaCu}$, es un modo de reconocer, en la experiencia vivida, una forma de conocimiento que puede develar verdades frente al fenómeno del cáncer vedadas desde otras perspectivas. Esto sucede gracias a 
que la experiencia, como un elemento constituyente del sujeto, conforma las condiciones de posibilidad para que el ser humano pueda acceder al mundo, es decir, pueda tener no solo una impresión sensitiva de este, sino también conocerlo empíricamente: elaborarlo, comprenderlo y, a partir de allí, construir otros tipos de experiencias. Así, la experiencia es un proceso de entendimiento donde finalmente cada sujeto termina convirtiéndose en el autor de su propia comprensión de mundo [21].

\section{Metodología}

\section{Diseño}

En este artículo se presentan los resultados del componente cualitativo de un estudio de métodos mixtos de triangulación convergente, cuyo componente cuantitativo fue un estudio de casos y controles, el cual buscaba establecer las causas del diagnóstico tardío del $\mathrm{CaCu}$ en mujeres de tres departamentos fronterizos de Colombia: Nariño, La Guajira y Amazonas, titulado: "Causas del diagnóstico tardío de cáncer de cuello uterino en tres departamentos del país y estrategias para implementar un programa para su control". Para los análisis presentados en este artículo, participaron mujeres de los departamentos de la Guajira y Nariño.

\section{Participantes y procedimientos}

Las participantes del estudio fueron aquellas mujeres a las que se logró acceder por medio de las instituciones prestadoras de servicios de salud (IPS) que hicieron parte del estudio. Así las cosas, en la Guajira se contó con participantes en los municipios de Riohacha, Manaure, Maicao, Uribía, San Juan del Cesar, Albania, El Molino, Barrancas, y Villanueva; y en Nariño, en Pasto, Tumaco, Ipiales y La Unión, municipios donde prestaban servicios las IPS vinculadas.

Para la fase cualitativa se convocaron mujeres del grupo de los casos, diagnosticadas con $\mathrm{CaCu}$ invasor con patología no mayor a 2 meses y menores de 69 años (límite de edad para tamizaje en Colombia), y mujeres del grupo de los controles, con una colposcopia/biopsia negativa o con neoplasia intraepitelial cervical 1 (NIC 1), no mayor a 2 meses, sin antecedente de patología cervical o histerectomía, que vivieran en el mismo municipio del caso, y con la misma edad o 5 años por encima o por debajo con respecto a las mujeres del grupo de los casos.

Las mujeres se captaron mediante las bases de datos de los laboratorios de patología, clínicas, hospitales y aseguradoras con las que se estableció un contacto previo. Una persona entrenada revisaba, de manera quincenal, las bases de datos, para identificar participantes que cumplieran los criterios de inclusión.
La recolección de información cualitativa se hizo entre octubre de 2016 y marzo de 2017.

Las mujeres que participaron en la fase cualitativa se convocaron posterior a la inclusión en el estudio cuantitativo, es decir, a todas las participantes se les había realizado un cuestionario previamente.

Para la selección de las mujeres para la fase cualitativa se tuvo en cuenta su disponibilidad e interés por participar. En el departamento del Amazonas no fue posible contactar participantes del estudio de casos y controles, debido al fallecimiento o cambio de domicilio de las mismas.

Se utilizaron dos técnicas de recolección de información: la entrevista, por su enorme potencial de maxificar significados, experiencias y creencias en la interacción "cara a cara" [22], y los grupos focales, que, por su pluralidad y variedad de opiniones, conducen a una riqueza de testimonios en un espacio de tiempo relativamente corto, siendo ideales para conocer el fenómeno de estudio en su contexto [23].

Las entrevistas y los grupos focales fueron conducidos por tres personas entrenadas en investigación cualitativa; fueron grabados en audio y, posteriormente, transcritos. Las entrevistas se llevaron a cabo en las casas de las mujeres, y los grupos focales, en una universidad y en la sede de una IPS.

En total se realizaron 9 entrevistas semiestructuradas, de aproximadamente una hora cada una; de estas, 5 se efectuaron en La Guajira (4 casos y un control), y 4 , en Nariño ( 3 casos y 1 control). En total se llevaron a cabo 3 grupos focales, de aproximadamente 2 horas cada uno; de estos, 2 se hicieron en Nariño (1 de casos y 1 de controles), en el que participaron 5 mujeres, y el otro se desarrolló con mujeres del grupo control en La Guajira, en el cual participaron 2 mujeres.

Las entrevistas realizadas a mujeres wayuu que no hablaban el español requirieron del apoyo de una intérprete y posteriormente fueron transcritas del wayuunaiki al español. Para ambas técnicas de recolección se utilizó una guía de preguntas semiestructurada, a partir de los siguientes ejes temáticos: 1) experiencias en el tamizaje de cuello uterino (citología), colposcopia, biopsia y otros; 2) acceso a los servicios de salud en términos geográficos, económicos y culturales; 3) problemas de oportunidad y calidad en la atención, y 4) experiencia con el tratamiento y la vivencia del cáncer.

\section{Análisis}

Se utilizó la técnica del análisis de contenido [24,25]. Dos investigadoras, de forma independiente, codificaron las entrevistas, teniendo en cuenta los ejes temáticos de la guía semiestructurada de preguntas; en este proceso se obtuvieron 191 códigos. Posteriormente, estos códigos, con sus respectivas frecuencias, se agruparon en cate- 
gorías y subcategorías, que emergieron en un proceso inductivo-deductivo.

Para facilitar el conteo y posterior agrupación de los códigos, se elaboraron tablas de frecuencia, utilizando hojas de cálculo de Excel®. En las filas se encontraban las categorías, subcategorías y códigos, y en las columnas se hacía el conteo de acuerdo con el actor entrevistado, es decir, los casos y controles de La Guajira y Nariño. Estas categorías fueron discutidas con una tercera investigadora hasta lograr consenso.

En este proceso, el conteo sirvió para reagrupar algunos códigos y robustecer las subcategorías. Surgieron tres categorías analíticas: 1) barreras en la atención en salud; 2) experiencia con el personal de salud y administrativo, y 3) la vivencia del cáncer.

\section{Consideraciones éticas}

El estudio fue aprobado por el Comité de Ética de la Facultad Nacional de Salud Pública de la Universidad de Antioquia, en el Acta 147 del 29 de julio de 2016.

Las participantes firmaron un consentimiento informado, y en el caso de las mujeres wayuu, el consentimiento fue explicado por un intérprete en wayuunaiki.

Se respetó su participación voluntaria y su confidencialidad, para lo que se utilizaron códigos que hacen imposible su identificación personal.

\section{Resultados}

Se entrevistó a 15 mujeres, 9 con cáncer invasor, 3 con diagnóstico de NIC 1, 2 con cervicitis crónica y 1 con pólipo endocervical de bajo grado, en los municipios de Manaure (1), Maicao (6), Uribía (1), Ipiales (2) y Pasto (5). En los demás municipios propuestos al inicio del estudio no se encontraron casos.

Las mujeres entrevistadas estaban entre los 43 y 65 años de edad, con una media de 51,2 y una desviación estándar de 6,27.

Aproximadamente, el 79 \% vivía en una cabecera municipal, y el resto, $21 \%$, en zona rural.

La mayoría $(53,3 \%)$ no tenía estudios o no terminó la primaria; el 13,3\% terminó primaria; el 26,7 \% concluyó el bachillerato, y una persona tenía título universitario.

Así mismo, la mayoría de las participantes (93,3\%) pertenecía al régimen subsidiado y solo una al régimen contributivo.

Adicionalmente, el $40 \%$ de las participantes eran indígenas.

No se encontraron diferencias en las experiencias relatadas de acuerdo a si las participantes tenían cáncer o una anormalidad citológica; por lo tanto, los resultados no se presentan por separado.

\section{Barreras en la atención en salud}

De acuerdo con los relatos de las participantes, se encontraron múltiples problemas burocráticos en la atención y el acceso a servicios, que van desde la toma de citología, hasta el tratamiento del cáncer. En este proceso, las mujeres relatan extensos itinerarios para acceder al servicio, así como trabas administrativas y demoras en la asignación de citas. Algunas participantes presentaron demoras para ser atendidas o para continuar con sus tratamientos, por problemas con las afiliaciones a las entidades promotoras de salud (EPS). También relatan problemas con los trámites administrativos, debido a las demoras para obtener autorización para citas médicas, exámenes diagnósticos, procedimientos, o tratamientos especializados, ante los cuales las EPS no brindaban soluciones oportunas.

La poca oferta de servicios versus la demanda, el cierre de servicios de salud y la falta de disponibilidad de convenios con IPS se convierten en una limitante para la atención y genera mayor fragmentación.

Cuando las mujeres lograban obtener las autorizaciones o remisiones a otros niveles de complejidad, encontraban demoras en la asignación de citas y otras trabas, como se aprecia en el siguiente testimonio:

Aquí es, ah, como yo no tengo contrato, eso es otro cuento; que si tiene contrato, las famosas EPS, entonces... ¡ay! Como yo no tengo contrato, señorita, señora, vaya a que le hagan otras cinco radioterapias. Así no es, están jugando con la vida de las personas (C005* - Participante con cáncer, Nariño).

Por las particularidades propias de los lugares donde vivían las mujeres, en su gran mayoría distantes de las capitales o cabeceras urbanas, obtener la autorización, citas médicas, o tratamientos implicaba asumir gastos de viaje, que en ocasiones debían ser cubiertos por las EPS; sin embargo, estos no siempre eran autorizados oportunamente, por lo cual ellas decidían asumir el traslado por cuenta propia:

Yo le dije a la empresa [EPS] que si me podría colaborar con los viáticos; entonces, ella me dijo que sí, pero que ahorita mismo no estaban dando viáticos, porque tenían problemas en la empresa; más, sin embargo, yo las amistades mías, por aquí hacían rifas y dos de mis hijos me colaboraban ahí, y ella [funcionaria de la EPS] me colaboraba bastante para la cuestión de los pasajes y eso, los viáticos (A006 Participante con cáncer, La Guajira).

Las consecuencias de la precariedad y baja oferta de servicios son múltiples, en especial para aquellas mujeres residentes en comunidades indígenas o municipios alejados, quienes deben trasladarse a otros departamentos o ciudades a realizarse exámenes diagnósticos,

Código asignado a las participantes para proteger su identidad. 
procedimientos y tratamiento para el cáncer. Lo anterior genera un alto costo, para su familia, en transporte y estadía para ellas y sus acompañantes; también ocasiona dificultades para encontrar una persona idónea que haga el acompañamiento y que además sepa desenvolverse en los servicios de salud; y en el caso de los wayuu, que hable español. Tales situaciones desmotivaron a algunas mujeres de recibir tratamiento fuera de sus ciudades o departamentos de origen:

Lo difícil es lo que te comento; además, ella [su hija] tiene un trabajo y hay días que no puede ir [a] acompañarme [a Barranquilla], porque ya trabaja. Mis otros hijos no tienen cédula, porque no son como ella. Además, ella conoce y sabe desenvolverse (A004 - Participante con cáncer, La Guajira).

Otro gasto que debe asumir la paciente o familia es el pago de las ayudas diagnósticas o consultas con profesionales, pues debido a la demora en la asignación de estos servicios, algunas mujeres se han visto en la necesidad de acceder a la atención de salud con sus propios recursos.

A mí me mandaron la biopsia para que yo me la hiciera y el médico me dijo: "Sí, yo te hago esa biopsia; eso se va a demorar para venir un mes y de ahí no sé cuándo llegue aquí...; pero si te la haces por aparte, se va a demorar 10 o 15 días, y enseguida te llegan los resultados; puedes llegar directamente para que te adelanten el tratamiento". Entonces, yo les comenté [a la familia] y ellos me dijeron: "Vamos a hacer lo posible para recoger, para pagar eso por aparte, para que te lo hagan rapidito, el tratamiento, porque ya tú estás mal". Me sentí con dolor, yo no dormía casi, me dormía en una silla y los pies arriba (A006 - Participante con cáncer, La Guajira).

\section{Experiencias con el personal de salud y administrativo}

Con relación al trato conferido por el personal de salud, se encontraron buenas y malas experiencias. Para algunas mujeres, el trato de médicos y especialistas fue el adecuado, al igual que las indicaciones posteriores a la cita, lo que genera confianza en los médicos:

En Pasto, sí, ¿para qué?, el doctor, muy buen ginecólogo; él no solo conmigo, con todas las pacientes; él es una buena persona, un buen médico (C011 - Participante con cáncer, Nariño).

Otras mujeres, por el contrario, tuvieron experiencias negativas en la atención, situación que genera desconfianza hacia los profesionales y las instituciones de salud. En este sentido, las participantes refieren negligencia del personal de salud para hacer la toma de citología, dar un diagnóstico oportuno u ordenar tratamiento adecuado, e incluso hablan de maltrato; pese a esto, las mujeres siguen el proceso de atención, según ellas, para conservar su vida.
Acerca de las razones por las cuales las mujeres reciben una "mala atención", una participante refiere que al sistema de salud solo le interesan las ganancias económicas, en detrimento de la salud de los usuarios:

Hay gente que contratan las EPS; lo que hacen, mira lo que hacen, el daño que hacen. ¿Por qué? Porque a ellas, lo que les interesa es economizar, economizar, economizar, economizar, eso es lo que les interesa a las EPS; por eso, contratan es una enfermera, ni una enfermera jefe; contratan es una auxiliar de enfermería, que lo único que les interesa es ganarse un sueldo y cumplir las metas que les colocan allá las EPS. A ellas lo que les interesa son las metas, promoción y prevención. ¿Cuántos pacientes usted atendió? ¿Cuántas citologías hizo en el día: 30, 20, 40? ¡Eso es suficiente! (C005 - Participante con cáncer, Nariño).

Otra afirma:

Fue un descuido muy duro de los médicos, porque si a mí me hubiera atendido a tiempo, no estuviera en esto... Pues el diagnóstico que dieron los médicos, fue un diagnóstico que tenía una infección urinaria en la cual no era así (C010 - Participante con cáncer, Nariño).

Un asunto que puede configurarse como una barrera o un facilitador en la atención de salud recibida por el personal es la comunicación que los profesionales logren entablar durante la prestación del servicio. Esto se evidencia en la explicación del diagnóstico, de la gravedad del caso y de los procedimientos y tratamientos a seguir, así como en la posibilidad que brinden a las mujeres de poder resolver las dudas que tengan sobre su proceso.

Al respecto, la mayoría de las mujeres entrevistadas expresaron haber recibido de parte del profesional tratante explicación de la enfermedad y del tratamiento necesario para lograr la curación, para lo cual el personal médico se esfuerza en comunicarse con la paciente de una manera que sea comprensible para ella. Esta situación, sin embargo, no es común a todos los casos, pues varias mujeres del estudio refirieron no haber recibido, del profesional de salud, explicaciones sobre su diagnóstico; no se sintieron escuchadas, ni tuvieron la comodidad de hacer preguntas acerca de lo que les estaba sucediendo o del tratamiento a seguir:

No, pues, eso no, no explican bien, [risas] ni tampoco

dejan preguntar (C003 - Participante con anormalidad, Nariño).

Esto último ayuda a comprender por qué cuando se indaga a las mujeres con diagnóstico de $\mathrm{CaCu}$ sobre lo que saben de su enfermedad, solo algunas alcanzan a mencionar que es una masa o tumor maligno, mientras que otras tienen ideas vagas sobre aquella. 
Investigadora: ¿Qué le dirías a un conocido, o dentro de la familia y comunidad, para que no se enfermen?

Participante: ¡No sé! ¿Y qué debería decirle? Si no conozco sobre la enfermedad que tengo; si la conociera, pudiera hablar (A005 - Participante con cáncer, La Guajira).

Algo similar sucede con el tratamiento recibido. Algunas mujeres aseguran no saber cuál y cómo fue el tratamiento recibido; o haber sido informadas del mismo, pero no por los profesionales de salud, sino gracias a la experiencia de personas conocidas:

\author{
Investigadora: ¿Conoces el examen que te hicieron? \\ Participante: No, no lo conozco. \\ Investigadora: ¿Qué tipo de control te hicieron? \\ Participante: No sé eso tampoco (A004 - Participante \\ con cáncer, La Guajira).
}

Otra participante dice:

Sí, lo había escuchado [el tratamiento]. Por parte de una señora que es vecina. Me decía por lo que pasaba y debía hacer (A005 - Participante con cáncer, La Guajira)

\section{La vivencia del cáncer}

Las mujeres relatan que la experiencia de tener cáncer les generó mucho sufrimiento, relacionado especialmente con la incertidumbre de la evolución de la enfermedad y el enfrentarse a la posibilidad de una muerte cercana.

[...] jes un golpe tan duro enterarnos de que tenemos cáncer! Es durísimo, para nuestra familia, para nuestros hijos, para nosotras mismas, es duro. Es bien duro uno saber que a uno ya le toca es vivir el día a día; yo ya no puedo, yo no puedo tener un futuro. Yo ya ni a mi hija la veo, en quince años no la puedo ni ver (C005 - Participante con cáncer, Nariño).

Los efectos secundarios del tratamiento hacen parte de la dificultad de esta experiencia. La pérdida de peso y especialmente la caída del cabello generan complejos en las mujeres, pues se sienten rechazadas por las personas con quienes interaccionan en su diario vivir, lo que afecta el desarrollo de sus actividades cotidianas.

[...] a ratos es como aburridor también. Uno a ratos se estresa. En mi casa, hay momentos que estoy bien, pero hay momentos que me estreso hartísimo. A mí, por lo menos, ¿sabe por qué? Por el gorro, porque yo después de tener un cabello tan lindo como el que yo tenía, quedar ahoritica como estoy, que yo ni salgo; entonces, uno, eso es lo que a mí me da, porque yo lo único que salgo es como se dice a [de] la tienda a mi casa, uno se estresa cuando está, después de uno estar enseñado a salir, enseñado a trabajar. Por lo menos ahorita yo no estoy trabajando, porque no puedo, porque lo que yo trabajo es en ventas ambulantes, trabajaba planchando, ¿no? Pero con todo esto que me ha tocado hacer, retirarme de mi trabajo, por lo menos ahorita me tocó retirarme de mis ventas, porque, pues, como es también, no, todas las personas, todas las personas no tienen un concepto bueno, ¿no? Ya lo ven a uno enfermo [y dicen:], “qué tendrá? Mejor alejémonos". A los hijos les van diciendo: "Vea, vayan a comprar a otra parte" y todo (C010 Participante con cáncer, Nariño).

Por otra parte, aquellas mujeres cuyo tratamiento requirió la resección de órganos reproductivos cuestionaron su feminidad, pues sienten que al perder una parte del cuerpo que las ha definido como mujeres, dicha feminidad podría verse alterada.

[...] la cirugía es muy dolorosa, que uno sinceramente se siente medio mujeres. Ustedes siguen siendo mujeres, yo no sigo siendo mujer, no tengo mi útero, no tengo nada. Me siento mutilada después de eso, de todas esas radioterapias [...] (C005 - Participante con cáncer, Nariño).

Y finalmente, enfrentarse a un diagnóstico de $\mathrm{CaCu}$ lleva a muchas de las mujeres a preocuparse por el dolor de su familia al enterarse del diagnóstico y al futuro de esta en caso de no lograr superar la enfermedad. Incluso, algunas refirieron no haber informado al grupo familiar de su situación, con el fin de evitarle sufrimiento.

[...] en el momento, lo único que yo pensé es: “¡Mi hijo!"; dije: "mi hijo se va a quedar solo, ¿qué hago?" [...] pues, entre todo, yo le pido tanto a mi Dios que me haga salir de todo esto, que me haga salir y que me dé salud por mis hijos... (C010 - Participante con cáncer, Nariño).

Sin embargo, cuando la mujer se anima a buscar apoyo en su grupo familiar, el común de las experiencias es que encuentren en él un apoyo emocional, motivacional, económico y de acompañamiento durante todo el proceso.

[...] mis hijos hombres me dicen que: "queremos verte bien, por eso estas en este proceso..." (A004 Participante con cáncer, La Guajira).

\section{Discusión}

En resumen, las participantes mencionaron numerosas barreras para acceder al diagnóstico y al tratamiento, como demora en la asignación y la autorización de citas, exámenes y procedimientos; el gasto de bolsillo ${ }^{\dagger}$ para traslados a ciudades capitales donde se brinda el servicio; dificultades para encontrar acompañamiento familiar fuera de sus comunidades de origen, y obstáculos idiomáticos en mujeres de comunidades indígenas. También se presentaron dificultades de comunicación efec-

$\dagger$ Pagos directos realizados por las personas en bienes y servicios del sector salud [26]. 
tiva con el personal de salud, lo que condujo a algunas participantes a no entender su diagnóstico o tratamiento, ni sentirse escuchadas. En el marco de un sistema de salud, en términos generales disfuncional, la vivencia del cáncer generó sufrimiento, rechazo de personas de su entorno social y despersonalización.

Con respecto al tiempo que tienen que esperar las mujeres para acceder a los servicios de salud (como citas médicas y pruebas diagnósticas) y para la entrega de los resultados en las pruebas de tamizaje, el dinero que deben invertir para acceder al diagnóstico o tratamiento, y la oportunidad para el inicio del tratamiento, varios estudios efectuados en diversas ciudades de Colombia coinciden con los resultados identificados en el presente estudio $[27,28]$. En estas investigaciones, las mujeres expresaron estos asuntos como razones de peso para no reclamar los resultados de sus pruebas diagnósticas, o no poder acceder al tratamiento para solucionar las lesiones, y con esto, no continuar el proceso de cuidado o atención de su salud [27].

Otro estudio, realizado por Garcés, Rubio y Ramos [9], y que coincide con lo descrito por Sánchez, Laza y Estupiñan [28], también señala que una de las principales barreras para el seguimiento, diagnóstico y tratamiento de anormalidades citológicas son las demoras que padecen las mujeres para que se les asignen las citas, problemas con las autorizaciones para acceder a la atención, trabas administrativas y los gastos de bolsillo que deben asumir para hacer uso de los servicios [9]. Adicionalmente, el desplazamiento excesivo que deben recorrer las mujeres para llegar a los lugares donde se les prestan los servicios es otro de los resultados identificados en el estudio y que coincide con lo señalado por Sánchez, Laza y Estupiñan [28].

Uno de los hallazgos de importancia para el estudio fue el desconocimiento que tenían las mujeres y sus familias para movilizarse por los diferentes servicios de salud, y lograr la atención requerida en cada uno de ellos. En el caso de las mujeres de La Guajira, este hecho no solo se acentuaba por no hablar español, sino también por la cantidad de trámites burocráticos para ser referidas a otros niveles de complejidad y a la fragmentación de los servicios. Otros investigadores reportan hallazgos similares: Puerta y VH Dover [29], desde el año 2006, encontraron en La Guajira grandes problemas que aún siguen vigentes, como la desarticulación de la salud pública y la salud individual, las dificultades de acceso, la baja calidad y oportunidad de los servicios, y el poco o nulo conocimiento de los usuarios acerca de la estructura del sistema. Es de anotar que, en el caso particular de las mujeres participantes de esta investigación, estas barreras generaron la vulneración del derecho a la atención en salud oportuna y de calidad.

Por otro lado, Cortés reporta experiencias de maltrato, inhumanidad y barreras de comunicación entre las mujeres wayuu y los trabajadores de la salud, pues entre estos últimos son pocos los que hablan el wayuunaiki [30]. También se reportan problemas burocráticos, como hacer largas filas y tener largas horas de espera para ser atendidas, sin priorizar a aquellas mujeres de rancherías alejadas del casco urbano, lo que acentúa las barreras geográficas [30]. Todos estos son hallazgos consistentes con los de este estudio.

Lo anterior demuestra fallas en las políticas, los programas y los proyectos de interculturalidad en salud que se han tratado de implementar en Colombia. Si bien existe un amplio marco normativo frente al tema y el "Plan Decenal de Salud", el "Modelo de acción integral territorial", entre otros, propenden por la transversalización del enfoque étnico diferencial, existe una disrupción entre la teoría y la práctica. Se podría pensar que, a corto y mediano plazo, la interculturalidad seguirá estando vigente en la agenda de salud pública, pero seguirá siendo un término conceptual [30].

En el caso de la interculturalidad con la población wayuu, existen actualmente varias estrategias, como la implementación de guías indígenas bilingües, la inclusión de personal de salud indígena, las adaptaciones locativas para que los pacientes y sus familias estén en un ambiente físico similar al de su hogar y facilitar la relación con los agentes de medicina tradicional; pese a esto, no se ha logrado una verdadera articulación y atención intercultural en salud [31]. Según Menéndez, esto puede obedecer a que en la atención en salud se han buscado establecer las diferencias de la medicina hegemónica con las "cosmovisiones indígenas", lo que resulta para la mayoría "incompatible". Lo recomendable sería, entonces, encontrar las similitudes, a partir de las cuales se puedan pensar las mejores vías para el desarrollo de procesos de aproximación [32].

Las barreras para el acceso oportuno a los servicios de salud en mujeres indígenas se pueden también analizar desde la teoría de la interseccionalidad. La "interseccionalidad" es una categoría desarrollada por los movimientos de feminismo negro en Estados Unidos, para explicar fenómenos asociados directamente con la injusticia social, experimentados por comunidades o sujetos a razón de la combinación de dos o más de sus condiciones esenciales, como la raza, el sexo, la etnia, la clase social, el tipo de funcionalidad, etc., creando de esta manera múltiples niveles de injusticia social [33]. Para el caso particular, nos encontramos frente a una población subalternizada, debido a su condición de mujer, indígena y pobre, pues si bien es sabido que el sistema de salud colombiano tiene serios problemas de acceso para gran parte de sus beneficiarios [34], esta situación se exacerba cuando se es mujer, pobre y de minoría étnica $[35,36]$, lo que además se acentúa en regiones alejadas del casco urbano o periferias. 
Este fenómeno se constituye en una especie de círculo vicioso, donde los vulnerables son constantemente vulnerados, con lo que se agudizan las brechas de inequidad y justicia social. Lo anterior se puede evidenciar en estudios como el de Bohren et al., según el cual mujeres con pocos recursos económicos recibieron peor tratamiento en salud y fueron humilladas por su pobreza, por vivir en zonas rurales o estar "sucias" o "desaliñadas" [37]. Y si, además de pobre, la mujer es indígena, la mala atención se padece doblemente, pues emergen expresiones de burla y rechazo respecto a sus concepciones y prácticas culturales [38]. Todo lo anterior pone en entredicho los principios de justicia, democracia y calidad que deben orientar los servicios de salud, así como la premisa según la cual los derechos de las mujeres, junto con los derechos de los niños, son uno de los indicadores más importantes de la veracidad de los derechos humanos [39].

Ahora bien, la interseccionalidad permite identificar lo imbricado de las relaciones de dominación, que oprimen y ponen en menor valía a sujetos o comunidades, según las características ya señaladas, lo que se expresa en una simultaneidad de tipos de opresión [40] que, como bien lo definió Foucault — citado por Retamal [41] - , son producto de una inadecuada gestión y regulación moral de las relaciones entre los seres humanos, haciendo imposible la práctica de libertad, y con ello, una existencia digna. Llama la atención que sea precisamente el mismo sistema de salud uno de los reproductores de estos mecanismos de dominación, pues naturaliza la invisibilización, la exclusión y el acallamiento de los grupos más vulnerados. La anterior se evidencia en la atención que reciben las mujeres indígenas de territorios fronterizos colombianos, donde los largos tiempos de espera, los extensos trámites burocráticos y la falta de adaptación cultural de los servicios se configuran en una serie de factores que ponen a la mujer que tiene un diagnóstico de $\mathrm{CaCu}$ a enfrentar una muerte casi segura.

Todos estos asuntos, que sobrepasan la comprensión de las mujeres y sus recursos económicos, familiares y comunitarios, deben ser demandados por la academia y la sociedad en general, pues una postura indiferente o cómplice de estos procesos de dominación sobre los más vulnerados no solo perpetúan, sino que además intensifican la violencia expresa o soterrada, padecida históricamente por estos grupos humanos [42].

Una limitación del estudio fue la necesidad del uso de intérpretes para comunicarnos con las mujeres wayuu. Sin embargo, se realizaron todos los esfuerzos necesarios para mantener la información lo más completa y fidedigna posible. Consideramos que, debido a la limitada evidencia científica acerca del seguimiento del $\mathrm{CaCu}$ en comunidades fronterizas con alta presencia de comunidades indígenas, esta investigación logra aportes de interés para los involucrados en la prevención y el control de este cáncer.

\section{Conclusiones y recomendaciones}

En conclusión, las mujeres con $\mathrm{CaCu}$ participantes en el estudio describieron barreras de diversa índole para el acceso oportuno al tratamiento, que se agudizan por características de base como la pobreza y la pertenencia a grupos étnicos, con lo que se convierte la supervivencia en una lucha diaria, que va más allá de las características propias de la enfermedad.

Finalmente, la atención integral del cáncer requiere de una gestión centrada en las necesidades específicas de las mujeres, que tenga en cuenta las diferencias culturales y sociales, para lograr mayor equidad en el acceso a programas de prevención y detección oportuna del $\mathrm{CaCu}$. Desde el sistema de salud se requieren cambios estructurales, que contribuyan al acceso real y efectivo a los servicios de salud de los departamentos relegados del territorio nacional. Se sugiere la creación de redes de atención pública que garanticen el acceso a todos los niveles de atención; por otro lado, se debe fortalecer la Atención Primaria en Salud en zonas de difícil acceso e incorporar promotores de salud que contribuyan a la comprensión intercultural.

\section{Agradecimientos}

A las mujeres participantes del estudio, en especial a aquellas que perdieron la batalla contra el cáncer.

\section{Declaración de fuente de financiación}

Este estudio fue financiado por Colciencias, contrato 628-2014, Universidad de Antioquia, Universidad de Nariño, Anas Wayuu EPSI (Empresa Promotora de Salud Indígena) y Secretaría de Salud Departamental del Amazonas.

\section{Declaración de conflictos de intereses}

Los autores no tienen ningún conflicto de interés para declarar.

\section{Declaración de responsabilidad}

Los puntos de vista expresados son responsabilidad de los autores y no de la institución en la que trabajan o de la fuente de financiación. 


\section{Contribución de los autores}

Diana Patricia Molina-Berrío: análisis e interpretación de datos, escritura del artículo y aprobación de la versión final del mismo. Se responsabiliza de todos los aspectos del trabajo.

Sara Milena Ramos-Jaraba: contribuciones sustanciales al diseño del estudio, recolección de la información, análisis e interpretación de datos, escritura del artículo y aprobación de la versión final del mismo. Se responsabiliza de todos los aspectos del trabajo.

Isabel Cristina Garcés-Palacio: concepción y diseño del estudio, interpretación de datos, contribuyó en la revisión crítica del artículo y en la aprobación de la versión final del mismo. Se responsabiliza de todos los aspectos del trabajo.

\section{Referencias}

1. World Health Organization. Cervical cancer [internet]. [citado 17 nov. 2019]. Disponible en: https://www.who.int/cancer/prevention/diagnosis-screening/cervical-cancer/en/

2. Ferlay J, Colombet M, Soerjomataram I, et al. Estimating the global cancer incidence and mortality in 2018: GLOBOCAN sources and methods. Int $J$ Cancer. 2019;144: 1941-53. DoI: https://doi. org/10.1002/ijc.31937

3. Pardo C, Cendales R. Cancer incidence estimates and mortality for the top five cancer in Colombia, 2007-2011. Colomb Med (Cali). 2018;49(1):16-22. DoI: http://dx.doi.org/10.25100/cm. v49i1.3596

4. Colombia. Congreso de la República. Ley 1384, Ley Sandra Ceballos, por la cual se establecen las acciones para la atención integral del Cáncer en Colombia (2010 abr. 19).

5. Colombia, Ministerio de Salud y Protección Social. Plan nacional para el control del cáncer en Colombia 2012-2020. Bogotá; 2012.

6. Colombia, Ministerio de Salud y Protección Social. Instituto Nacional de Cancerología. Modelo para el Control del Cáncer en Colombia. Bogotá, 2006.

7. Colombia, Ministerio de Salud y Protección Social, Instituto Nacional de Cancerología. Plan Decenal para el Control del Cáncer 2012-2021 [internet]; 2012 [citado 2010 feb. 10]. Disponible en: https://www.minsalud.gov.co/sites/rid/Lists/BibliotecaDigital/ RIDE/IA/INCA/plan-nacional-control-cancer-2012-2020.pdf

8. Cuenta de Alto Costo. Día Mundial de la Prevención del Cáncer de Cuello Uterino [internet]. Boletín de Información Técnica Especializada [internet]. 2018 mar. 26 [citado 2019 nov. 14]; 4(4). Disponible en: https://cuentadealtocosto.org/site/investigaciones/ dia-mundial-de-la-prevencion-del-cancer-de-cuello-uterino/

9. Garcés-Palacio IC, Rubio-León DC, Ramos-Jaraba SM. Barreras y facilitadores del sistema de salud relacionadas con el seguimiento de anormalidades citológicas, Medellín-Colombia. Rev Gerenc Polit Salud. 2014;13(27): 200-11. Dor: https://doi.org/10.11144/ Javeriana.rgyps 13-27.bfss

10. Valencia-Arredondo M. Otra cara del cáncer cervicouterino: una mirada desde la clase social y la cultura de género [Tesis de doctorado]. [Medellín:] Universidad de Antioquia; 2013.

11. Aranguren Pulido LV, Burbano Castro JH, González JD, et al. Barreras para la prevención y detección temprana de cán- cer de cuello uterino: revisión. Investig Enferm Imagen Desarr. 2017;19(2):129-43. DOI: http://dx.doi.org/10.11144/Javeriana. ie19-2.bpdt

12. Egurrola-Pedraza JA, Gómez-Wolff LR, Ossa-Gómez CA, et al. Diferencias en supervivencia debidas al aseguramiento en salud en pacientes con cáncer de mama atendidas en un centro oncológico de referencia en Medellín, Colombia. Cad. Saúde Pública. 2018;34(12):e00114117. DOI: http://dx.doi.org/10.1590/0102$311 \times 00114117$

13. Ramos-Jaraba SM, Carrillo-Pineda M. Significados que construyen mujeres afrodescendientes frente al cáncer de mama y cuello uterino, atendidas en Medellín, Colombia. Univ. Salud. 2018;20(2):111-120. DoI: http://dx.doi.org/10.22267/ rus. 182002.115

14. Rodríguez-Triana DR., Benavides-Piracón JA. Salud y ruralidad en Colombia: análisis desde los determinantes sociales de la salud. Rev Fac Nac Salud Pública [internet]. 2016; 34(3): 359-71. DOI: http://dx.doi.org/10.17533/udea.rfnsp.v34n3a10.

15. Colombia, Ministerio de Salud y Protección Social, Instituto Nacional de Cancerología. Boletín Servicios oncológicos en Colombia [internet]; 2016 [citado 2019 nov. 14]. Disponible en: https:// www.cancer.gov.co/files/libros/archivos/\%20Servicios\%200ncologicos\%20Bolet\%C3\%ADn.pdf

16. Puerta Silva C. Roles y estrategias de los gobiernos indígenas en el sistema de salud colombiano. Rev Colomb Antropol. 2004;40:85121

17. Bedoya-Murillo C, García-Restrepo D, Pérez-Collante Y. Barreras en el acceso a servicios de salud en pacientes con diagnósticos de enfermedades oncológicas en Colombia: Revisión sistemática de la literatura. [Monografía de especialización]. [Medellín:] Universidad CES; 2016.

18. Garcés IC, Rubio DC, Scarinci IC. Factores asociados con el tamizaje de cáncer de cuello uterino en mujeres de nivel socioeconómico medio y bajo en Bogotá, Colombia. Rev Fac Nac Salud Pública. 2012;30(1):7-16.

19. Programa Regional Salud de los Pueblos Indígenas. Prestación de servicios de salud en zonas con pueblos indígenas. Quito: Canadian International Development Agency (CIDA); 2009.

20. Wiesner C, Cendales R, Murillo R, et al. Seguimiento de mujeres con anormalidad citológica de cuello uterino, en Colombia. Rev. Salud Pública [internet]. 2010 [citado 2020 ago. 25]; 12(1):1-13. Disponible en: http://www.scielo.org.co/scielo.php?script=sci arttext\&pid=S0124-00642010000100001\&lng=en

21. Amengual G. El concepto de experiencia: de Kant a Hegel. Tópicos. 2007;(15):5-30.

22. Taylor S, Bogdan R. Introducción a los métodos cualitativos de investigación. 2. ${ }^{a}$ ed. Barcelona: Paidós; 1987.

23. Hamui-Sutton A, Varela-Ruiz M. La técnica de grupos focales. Investigación Educ. Médica [internet]. 2013 [citado 2020 feb 28]; 2(5): 55-60. Disponible en: http://www. scielo.org. $\mathrm{mx} / \mathrm{scielophp}$ ? script $=$ sci_arttext\&pid $=$ S200750572013000100009\&lng=es

24. Krippendorff K. Content Analysis: An Introduction to Its Methodology. 2nd ed. Thousand Oaks, ca: Sage Publications; 2004.

25. Downe-Wamboldt B. Content analysis: Method, applications, and issues. Health Care Women Int. 1992;13(3):313-21. DoI: https:// doi.org/10.1080/07399339209516006

26. Colombia, Ministerio de Salud y Protección Social. Cifras financieras del sector salud. Gasto en salud de Colombia: 2004-2011. 
Boletín bimestral No. 2. Bogotá: Dirección de Financiamiento Sectorial, Ministerio de Salud y Protección Social; 2014.

27. López-Castillo CA, Calderón MA, Gonzáles MM. Conocimientos, actitudes y prácticas respecto al cáncer de cuello uterino de mujeres entre 14 y 49 años de un barrio de la comuna 10 de la ciudad de Armenia, Colombia. Rev. Méd. Risaralda. 2013;19(1):14 20

28. Sánchez G, Laza C, et al.Barreras de acceso a los servicios de salud: narrativas de mujeres con cáncer de mama en Colombia. Rev. Fac. Nac. Salud Pública 2014;32(3):305-13.

29. Puerta-Silva C, VH Dover R. Desarrollo de un modelo de interlocución para el sistema de salud basado en el caso de La Guajira colombiana. Rev. Salud Pública [internet]. 2007 [citado 2020 ago. 25]; 9(3):353-68. Disponible en: http://www. scielo.org.co/scielo.php?script $=$ sci arttext\&pid $=\mathrm{S} 0124$ 00642007000300004\&lng=en

30. Cortés C. Representaciones sociales del cáncer de cuello uterino en mujeres wayuu, en prestadores de servicios de salud y en tomadores de decisión del municipio de Uribia del departamento de La Guajira, Colombia [Ph.D Public Health]. [Bogotá, D.C.]: Universidad Nacional de Colombia; 2016.

31. Duarte-Gómez M, Gómez Dantés O, Alegría O, et al. Un modelo de atención en salud al pueblo wayúu en la frontera colombovenezolana. Rev Panam Salud Pública. 2011;30(3):272-8.

32. Menéndez EL. Salud intercultural: propuestas, acciones y fracasos. Ciênc Saúde Coletiva. 2016;21(1):109-18. Dor: https://doi org/10.1590/1413-81232015211.20252015

33. La Barbera, M. Interseccionalidad, un "concepto viajero": orígenes, desarrollo e implementación en la Unión Europea. Interdisciplina. 2016;4(8):105-22. DoI: http://dx.doi.org/10.22201/ ceiich.24485705e.2016.8.54971

34. Ayala J. La salud en Colombia: más cobertura pero menos acceso. Documentos de trabajo sobre economía regional \#204 [internet]. Cartagena: Banco de la República, Centro de Estudios Econó- micos Regionales (CEER); 2014 [citado 2019 oct. 19]. Disponible en: http://www.banrep.gov.co/docum/Lectura_finanzas/pdf/ dtser_204.pdf

35. Ewig C, Hernández A. Gender equity and health sector reform in Colombia: Mixed state-market model yields mixed results. Soc Sci Med. 2009;68(6):1145-52. DoI: http://dx.doi.org/10.1016/j. socscimed.2008.12.039

36. Arrivilla Quintero M, Piñeros Jiménez J. Reconocimiento de las diversidades y del pluralismo en el mundo globalizado. Condición para una praxis ética en salud pública. En: Bioética y salud pública. Encuentros y tensiones. Bogotá: Universidad Nacional de Colombia; 2007. pp. 211-28.

37. Bohren MA, Vogel JP, Hunter EC, et al. The mistreatment of women during childbirth in health facilities globally: A mixedmethods systematic review. PLoS Med. 2015;12: e1001847; discussion e. Dor: https://doi.org/10.1371/journal.pmed.1001847

38. Ariza J, Hernández M. Equidad de etnia en el acceso a los servicios de salud en Bogotá, Colombia, 2007. Rev. Salud Pública [internet]. 2008 [citado 2019 oct. 19]; 10(Supl. 1):58-71. Disponible en: https://www.scielosp.org/pdf/rsap/2008.v10suppl1/58-71/es

39. Naciones Unidas. Los derechos de la mujer son derechos humanos [internet]; 2014 [citado 2019 oct. 19]. Disponible en: https:// www.ohchr.org/Documents/Publications/HR-PUB-14-2_SP.pdf

40. Viveros Vigoya M. La interseccionalidad: una aproximación situada a la dominación. Debate Feminista. 2016;52:1-17. DoI: https://doi.org/10.1016/j.df.2016.09.005

41. Retamal C. Consideraciones sobre poder y dominación en la formación de la subjetividad moderna. Universum. 2008;23(2):16683. Dor: http://dx.doi.org/10.4067/S0718-23762008000200010

42. Bidaseca K. Nuevas exhalaciones II. La intersectorialidad enlas marcas indelebles de los cuerpos femeninos subalternos para una política de la memoria. Revista Educación y Humanismo. 2013;15(24):38-53. 